\title{
Tin Whiskers: Electron Microscopy and EBSD Characterization
}

\author{
D.F. Susan, J.R. Michael, R.P. Grant, and W.G. Yelton \\ *Sandia National Laboratories, Albuquerque, NM
}

Tin (Sn) whiskers are conductive Sn filaments that grow from Sn-plated surfaces, such as surface finishes on microelectronics. The phenomenon of Sn whiskering has become a major concern in recent years due to requirements for lead $(\mathrm{Pb})$-free soldering and surface finishes in commercial electronics. In general, pure $\mathrm{Sn}$ finishes are more prone to whisker growth than their $\mathrm{Sn}-\mathrm{Pb}$ counterparts and high profile failures due to whisker formation (causing short circuits) have been documented.[1] Although a full explanation of the whisker growth mechanism has yet to be developed, much has been learned about whiskers, largely through SEM and related microscopy techniques.[2]

First, whiskers grow from their base, not from their tip. Evidence for this is shown in Fig. 1, which displays time-lapse SEM photomicrographs of whisker growth. The sample consists of electroplated $\mathrm{Sn}$ on a commercially pure $\mathrm{Cu}$ substrate (sheet). The whisker tip morphology remains unchanged throughout the growth process. Second, whisker diameters are typically on the order of one to a few microns, generally corresponding to the grain size of the underlying Sn film. Third, whiskers are usually single crystals. This is true even if the whiskers are bent or kinked, as shown in Fig. 2. The EBSD patterns obtained from this kinked whisker were unchanged along the length of the whisker and on either side of the kinks.

It is almost universally accepted that whiskers grow due to the driving force of a compressive stress in the Sn film. The whisker growth rate can be increased significantly by aplying an external compressive stress in the plane of the film and very fast whisker growth rates have been observed.[3] Figure 3 exhibits whisker growth measurements obtained from time-lapse SEM analysis over a period of about a week. An incubation period of 4-5 days (after Sn plating) was observed for the samples in this study. For straight whiskers, growth rates of about 3 microns per day were determined, with the rate tapering off slightly over time. A decreasing growth rate might be expected for a diffusional process, albeit with anomalously fast diffusion overall. Note also that the length measurements in Fig. 3 were obtained from (projected) SEM images. Thus, the true angle of the whisker with respect to the film surface is unknown and the absolute lengths may not be correct. However, it is believed that the growth rate measurements are good order-of-magnitude estimates, with the projection effect possibly accounting for the wide scatter in the data. Preliminary analysis with laser confocal scanning microscopy (LCSM) has been performed (not shown here) to determine growth angles, and therefore, true whisker lengths. Finally, Fig. 3b shows whisker length measurements over time for kinked whiskers. The times at which kinks were first observed are noted in the plot. The kink process almost always results in a significant reduction in growth rate, if not a complete termination of growth. Again, the SEM projection effect could be coming into play but, based on the predominance of this observation, it is believed that the kink process is important in relation to the whisker growth mechanism. That is, if the underlying process and crystallography for kinks (and whisker growth cessation) could be determined, it may be useful for understanding the conditions that actually make whisker growth more likely.

* Sandia is a multiprogram laboratory operated by Sandia Corporation, a Lockheed Martin Company, for the US Dept. of Energy's national Nuclear Security Administration under contract DE-AC04-94AL85000.

[1] NASA Goddard Space Flight Center Tin Whisker Homepage, website http://nepp.nasa.gov/whisker/ 
[2] G.T. Galyon and L. Palmer, IEEE Trans. Elec. Packaging Manuf., 28, (1), 17-30, 2005.

[3] R.M. Fisher et al., Acta Met., Vol. 2, 368-373, 1954.
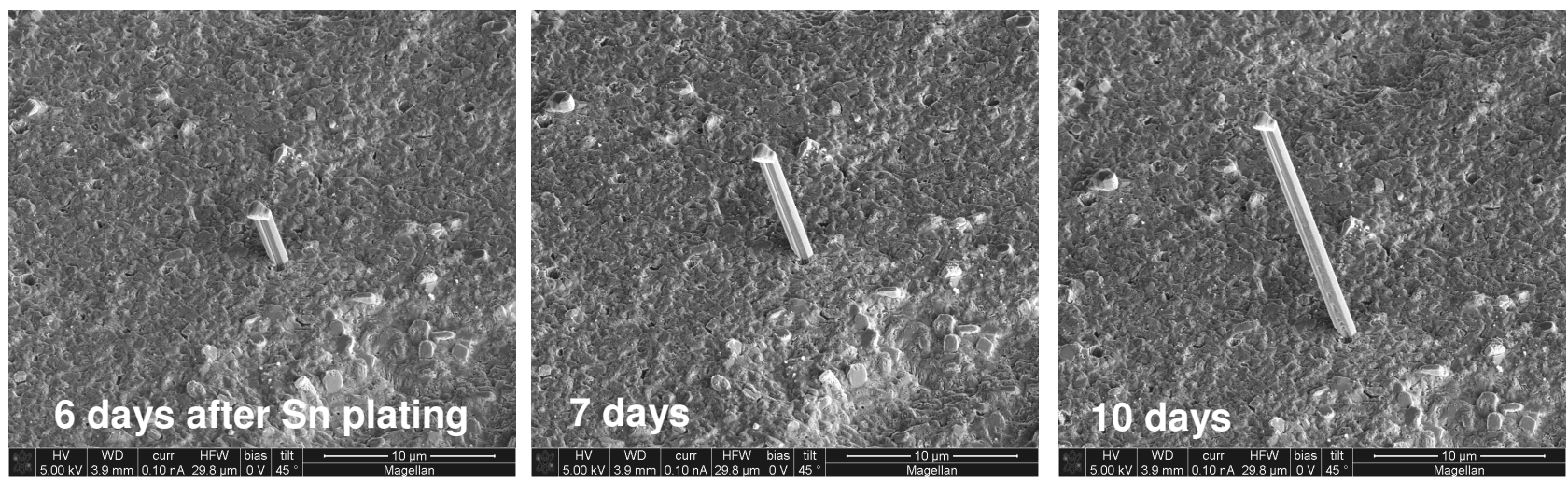

Fig. 1. SEM photomicrographs of Sn whisker growth. The tip morpholoogy remains unchanged, with a so-called "cap grain" on top.
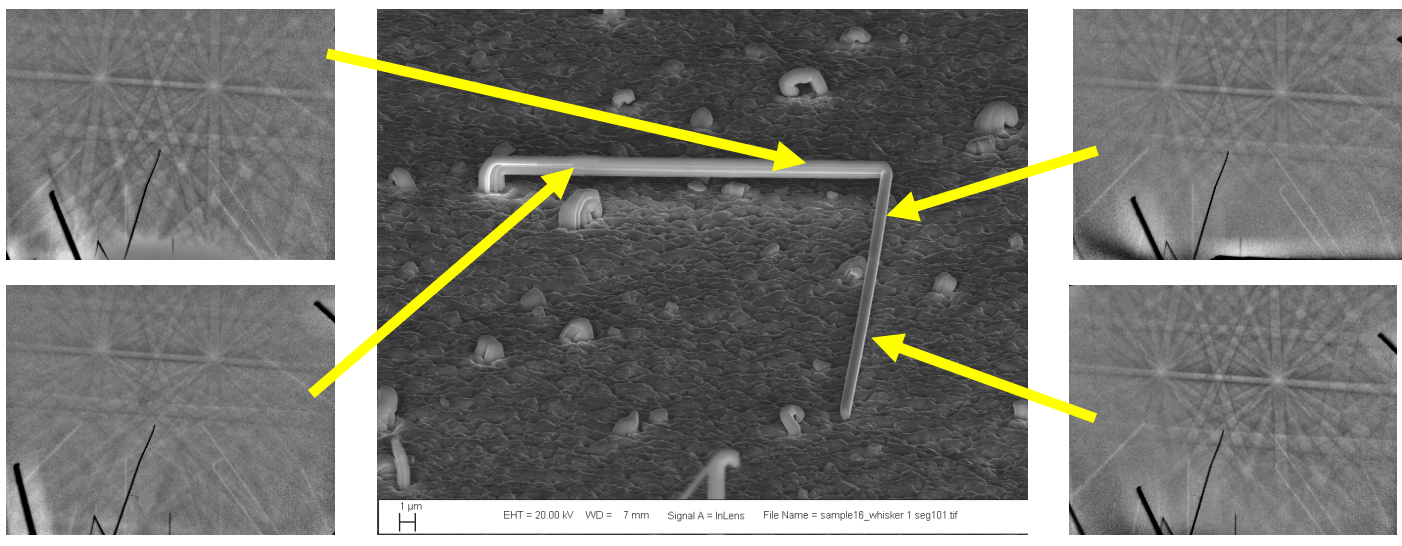

Fig. 2. A Sn whisker with two kinks. The EBSD patterns remain unchanged showing that the whisker is single crystal with the same crystallographic orientation throughout.
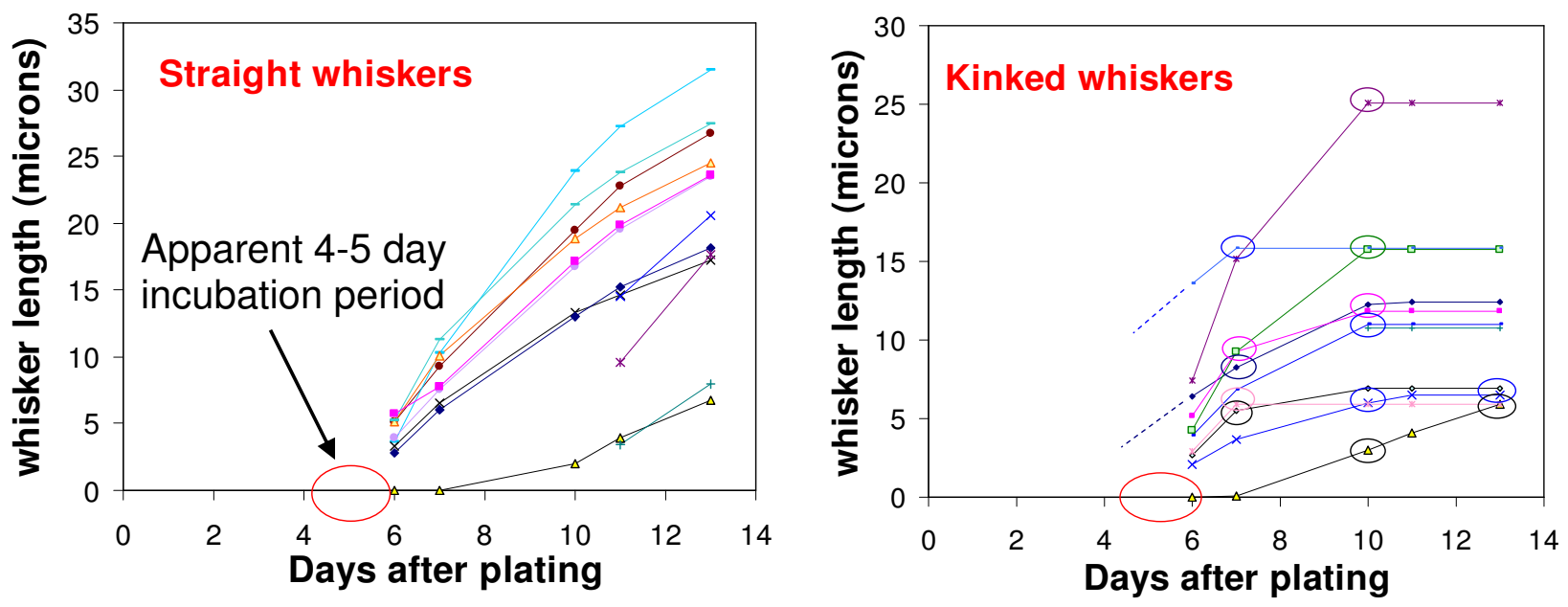

Figs. 3a and 3b. Whisker lengths vs. time, obtained by in-situ SEM of more than 20 Sn whiskers. Ovals show the times when kinks were first observed, after which growth slows down or stops. 